\title{
Schatten p-classes and asymptotics for eigenvalues of positive integral operators on the sphere
}

\author{
Ana C. Piantella, $\quad$ Mario H. de Castro, \\ Faculdade de Matemática, FAMAT-UFU, \\ 38408-100, Uberlândia, MG \\ E-mail: piantella@famat.ufu.br, mariocastro@famat.ufu.br \\ V. A. Menegatto \\ ICMC/USP \\ 13566-590, São Carlos, SP \\ E-mail: menegatt@icmc.usp.br.
}

\begin{abstract}
We obtain decay rates for eigenvalues and singular values of positive integral operators generated by square integrable kernels on the unit sphere in $\mathbb{R}^{m+1}, m \geq 2$, under assumptions on both, certain derivatives of the kernel and the integral operators generated by such derivatives. This type of problem is common in the literature but the assumptions are usually defined using standard differentiation in $\mathbb{R}^{m+1}$. The rates we present depend on both, the differentiability order used to define the smoothness conditions and the dimension $m$. Some of them are shown to be optimal. The results we show here are published in [2] except Theorem 2.5 which extends the main result from there.
\end{abstract}

Palavras-chave: Sphere, spherical harmonics, integral operators, eigenvalues, singular values, decay rates, positive definite kernels, Laplace-Beltrami derivative.

\section{Introduction}

Let $m \geq 2$ be an integer and $S^{m}$ be the unit sphere in $\mathbb{R}^{m+1}$. Let $d x$ be the usual volume element on $S^{m}$ and $L^{2}\left(S^{m}\right)$ the Hilbert space of all square-integrable complex functions on $S^{m}$ endowed with the inner product

$$
\langle f, g\rangle_{2}:=\frac{1}{\sigma} \int_{S^{m}} f(x) \overline{g(x)} d x, \quad f, g \in L^{2}\left(S^{m}\right)
$$

and the derived norm $\|\cdot\|_{2}$, the normalization constant being defined by $\sigma:=\int_{S^{m}} d x$.

We deal with integral operators defined by

$$
\mathcal{K}(f)=\int_{S^{m}} K(\cdot, y) f(y) d y,
$$

in which the generating kernel $K: S^{m} \times S^{m} \rightarrow \mathbb{C}$ is an element of $L^{2}\left(S^{m} \times S^{m}\right)$. In this case, (1.1) defines a compact operator on $L^{2}\left(S^{m}\right)$.

If $K$ is positive definite in the sense that

$$
\int_{S^{m}} \int_{S^{m}} K(x, y) f(x) \overline{f(y)} d x d y \geq 0, \quad f \in L^{2}\left(S^{m}\right)
$$

\footnotetext{
*Partially supported by CNPq, Grant \#475320/2013-1 and PROPP-UFU
} 
then $\mathcal{K}$ becomes a self-adjoint operator and the standard spectral theorem for compact and self-adjoint operators is applicable and we can write

$$
\mathcal{K}(f)=\sum_{n=0}^{\infty} \lambda_{n}(\mathcal{K})\left\langle f, f_{n}\right\rangle_{2} f_{n}, \quad f \in L^{2}\left(S^{m}\right),
$$

in which $\left\{\lambda_{n}(\mathcal{K})\right\}$ is a sequence of nonnegative reals (possibly finite) decreasing to 0 and $\left\{f_{n}\right\}$ is an $\langle\cdot, \cdot\rangle_{2}$-orthonormal basis of $L^{2}\left(S^{m}\right)$. The numbers $\lambda_{n}(\mathcal{K})$ are the eigenvalues of $\mathcal{K}$ and the sequence $\left\{\lambda_{n}(\mathcal{K})\right\}$ takes into account possible repetitions implied by the algebraic multiplicity of each eigenvalue. The positive definiteness of $K$ means nothing but the positivity of the integral operator $\mathcal{K}$. Since it relates to the inner product above, it is a common sense to call it $L^{2}$-positive definiteness.

We observe that the addition of continuity to $K$ implies that $\mathcal{K}$ is also trace-class (nuclear) $([5,8,9])$, that is,

$$
\sum_{f \in B}\left\langle\mathcal{K}^{*} \mathcal{K}(f), f\right\rangle_{2}^{1 / 2}<\infty
$$

whenever $B$ is an orthonormal basis of $L^{2}\left(S^{m}\right)$. In particular, it follows from Mercer's Theorem [3] that nuclearity is equivalent to

$$
\sum_{n=1}^{\infty} \lambda_{n}(\mathcal{K})=\int_{S^{m}} K(x, x) d x<\infty,
$$

in that case. As so we can extract the most elementary result on decay rates for the eigenvalues of such operators, namely,

$$
\lambda_{n}(\mathcal{K})=o\left(n^{-1}\right) .
$$

The notation above means that the sequence of eigenvalues approximates to zero "faster" than the sequence $\left\{n^{-1}\right\}$, i.e., $\lim _{n \rightarrow \infty} n \lambda_{n}(\mathcal{K})=0$.

If the integral operator $\mathcal{K}$ is compact but not self-adjoint then decay rates for the singular values of the operator becomes the focus. If $T$ is a compact operator on $L^{2}\left(S^{m}\right)$, its eigenvalues can be ordered as $\left|\lambda_{1}(T)\right| \geq\left|\lambda_{2}(T)\right| \geq \cdots \geq 0$, counting multiplicities ([13]). The singular values of $T$ are, by definition, the eigenvalues of the compact, positive and self-adjoint operator $|T|:=\left(T^{*} T\right)^{1 / 2}$. The sequence $\left\{s_{n}(T)\right\}$ of singular values of $T$ can also be ordered in a decreasing manner, with repetitions being included according to their multiplicities as eigenvalues of $|T|$. That being the case, the classical Weyl's inequality ([9, p.52])

$$
\Pi_{j=1}^{n}\left|\lambda_{j}(T)\right| \leq \Pi_{j=1}^{n} s_{j}(T), \quad n=1,2, \ldots,
$$

provides the convenient bridge between eigenvalues and singular values. We remark that the inequality characterizing the traceability of a compact non self-adjoint operator $T$ on $L^{2}\left(S^{m}\right)$ reduces itself to

$$
\sum_{n=1}^{\infty} s_{n}(T)<\infty
$$

Classical references on eigenvalues and singular values distribution of compact operators on Banach spaces are $[13,18]$.

The idea of nuclearity can be extended as follows. For $p>0$ we say that a compact operator $T$ belongs to the Schatten $p$-class $\mathcal{S}_{p}$ if

$$
\sum_{n=1}^{\infty}\left(s_{n}(T)\right)^{p}<\infty
$$

For $p \geq 1, \mathcal{S}_{p}$ is a Banach space with the norm

$$
\|T\|_{p}:=\left(\sum_{n=1}^{\infty}\left(s_{n}(T)\right)^{p}\right)^{1 / p}
$$


In particular, $\mathcal{S}_{2}$ coincides to the space of Hilbert-Schmidt operators. See [9] for more information on this subject.

The object of study in this paper is the analysis of decay rates for the sequence $\left\{\lambda_{n}(\mathcal{K})\right\}$ under additional assumptions on the kernel $K$ and certain derivatives of it. Results of this very same nature can be found in many references abroad and not necessarily in the context discussed here. In particular, we mention the use of integrated Hölder assumptions on $K$ in $[1,4,15]$ and of Lipschitz type in $[6,7]$. As a matter of fact, the ideas of some of these cited papers have their origin in $[12,14]$ where similar kernels have been studied. The intention here is to present the results obtained in [2] in which we use the Laplace-Beltrami derivative to define the basic assumptions needed (we also extend one of them). As far as we know, this approach is new and fits more properly since such derivative is a concept genuinely spherical, having many interesting properties and applications in connection with Approximation Theory (see [16, 17] and references therein) and other areas as well. By the way, the Laplace-Beltrami derivative was introduced by W. Rudin in [19] and further developed by Wherens in [20, 21], but in the case $m=2$ only. The general case is fully discussed in the survey-like paper [17].

\section{Statement of the main results}

The Laplace-Beltrami derivative is a variation of the usual derivative on $S^{m}$ when, in the definition of the later, one replaces the usual translation operator with the spherical shifting operator, which is defined by the formula

$$
T_{\epsilon}^{m}(f)(x):=\frac{1}{\sigma_{m-1}\left(1-\epsilon^{2}\right)^{(m-1) / 2}} \int_{x \cdot y=\epsilon} f(y) d y, \quad x \in S^{m} .
$$

Here, $\epsilon \in(-1,1)$, " ." is the usual inner product of $\mathbb{R}^{m+1}$ and $d y$ denotes the measure element of the rim $\left\{y \in S^{m}: x \cdot y=\epsilon\right\}$ of the spherical cap $\left\{y \in S^{m}: x \cdot y \geq \epsilon\right\}$. If we write $\Delta_{\epsilon}:=I-T_{\epsilon}^{m}$, in which $I$ denotes the identity operator, a function $f \in L^{2}\left(S^{m}\right)$ is said to be differentiable in the sense of Laplace-Beltrami if there exists $\mathcal{D} f \in L^{2}\left(S^{m}\right)$ such that

$$
\lim _{\epsilon \rightarrow 1^{-}}\left\|(1-\epsilon)^{-1} \Delta_{\epsilon}(f)-\mathcal{D} f\right\|_{2}=0 .
$$

The symbol $\|\cdot\|_{2}$ above stands for the usual norm of $L^{2}\left(S^{m}\right)$. The function $\mathcal{D} f$ is then called the Laplace-Beltrami derivative of $f$. Higher order derivatives are defined by the formulas $\mathcal{D}^{1}=\mathcal{D}$ and

$$
\mathcal{D}^{r}:=\mathcal{D}^{1} \circ \mathcal{D}^{r-1}, \quad r=2,3, \ldots
$$

We now introduce basic Sobolev-type spaces for functions on $S^{m}$.

Definition 2.1. The space of all complex functions on $S^{m}$ which are differentiable, up to order $r$, in the sense explained above, will be denoted by $W_{2}^{r}$.

The operator $\mathcal{D}^{r}$ is a multiplier operator in the sense we now explain. If $\mathcal{H}_{n}^{m+1}$ is the space of all $n$-th degree spherical harmonics in $m+1$ variables then $\mathcal{H}_{n}^{m+1}$ is a subset of $W_{2}^{r}$ and

$$
\mathcal{D}^{r} Y=\frac{n^{r}(n+m-1)^{r}}{m^{r}} Y, \quad Y \in \mathcal{H}_{n}^{m+1} .
$$

It acts like a self-adjoint operator on $W_{2}^{r}$, that is,

$$
\left\langle\mathcal{D}^{r} f, \bar{g}\right\rangle_{2}=\left\langle f, \overline{\mathcal{D}^{r} g}\right\rangle_{2}, \quad f, g \in W_{2}^{r} .
$$

For more information on the Laplace-Beltrami derivative we refer the reader to [17] and references mentioned there. In particular, one can find explained there a connection among the 
Laplace-Beltrami derivative, the usual derivative for functions on $S^{m}$ and the so-called $r$-th spherical modulus of smoothness.

The action of the Laplace-Beltrami derivative on kernels is done separately: we keep one variable fixed and differentiate with respect to the other. The symbol $\mathcal{D}_{y}^{r} K$ will indicate the $r$-th order derivative of a kernel $K$ with respect to the variable $y$ (we will never differentiate with respect to the first variable $x$ ). For $r \in \mathbb{Z}_{+}$, we find convenient to introduce the following notation

$$
K_{0, r}(x, y):=\mathcal{D}_{y}^{r} K(x, y), \quad x, y \in S^{m},
$$

to abandon the derivative symbols. The integral operator associated with $K_{0, r}$ will be written as $\mathcal{K}_{0, r}$. At this point, it is convenient to introduce Sobolev-type spaces for kernels in a formal way.

Definition 2.2. A kernel $K \in L^{2}\left(S^{m} \times S^{m}\right)$ belongs to $W_{2}^{r}$ when $K(x, \cdot) \in W_{2}^{r}, x \in S^{m}$ a.e..

We are ready to describe the main results of the paper. We emphasize that all the results take for granted the ordering on either the eigenvalues or singular values mentioned before. At first, we will prove a theorem without the $L^{2}$-positive definiteness assumption on $K$ and obtain a decay rate for the sequence of singular values of $\mathcal{K}$.

Theorem 2.3. Let $r$ be a positive integer at least $(m+1) / 2, K$ an element of $W_{2}^{r}$ and $p \in(m+1,2 r+1]$. If $\mathcal{K}_{0, r}$ is bounded then

$$
s_{n}(\mathcal{K})=o\left(n^{-1-(2 r+1-p) / m}\right) .
$$

We observe that the fact that the derivatives $\mathcal{D}_{y}^{r} K(x, \cdot)$ exist for $x \in S^{m}$ a.e. does not imply that $\mathcal{K}_{0, r}$ is a bounded operator. As so, the assumption on $\mathcal{K}_{0, r}$ in Theorem 2.3 is reasonable. Clearly, the smaller the parameter $p$, the better the estimate.

The next two results incorporate $L^{2}$-positive definiteness as an assumption. As so, they describe decay rates for the eigenvalues of $\mathcal{K}$ under certain hypotheses on either $K_{0, r}$ or $\mathcal{K}_{0, r}$.

Theorem 2.4. Let $K$ be a $L^{2}$-positive definite kernel in $W_{2}^{r}$. If $K_{0, r}$ belongs to $L^{2}\left(S^{m} \times S^{m}\right)$ then

$$
\lambda_{n}(\mathcal{K})=o\left(n^{-1 / 2-2 r / m}\right) .
$$

If we assume $\mathcal{K}_{0, r}$ belongs to some Schatten $p$-class instead the basic assumption in Theorem 2.4 then we obtain the following improvement on the previous decay rate.

Theorem 2.5. Let $K$ be a $L^{2}$-positive definite kernel in $W_{2}^{r}$. If $\mathcal{K}_{0, r} \in \mathcal{S}_{p}$ then

$$
\lambda_{n}(\mathcal{K})=o\left(n^{(-1 / p)-(2 r / m)}\right)
$$

We state the next result because in this case we can show the decay rate are the best possible.

Corollary 2.6. Let $K$ be a $L^{2}$-positive definite kernel in $W_{2}^{r}$. If $\mathcal{K}_{0, r}$ is trace-class then

$$
\lambda_{n}(\mathcal{K})=o\left(n^{-1-2 r / m}\right) .
$$

To close the section, we would like to inform the reader that the results above resemble those proved in $[8$, p.120] and $[10,11]$ for the case of an interval. As a matter of fact, one can interpret Theorem 2.4 and Corollary 2.6 as spherical versions of some of the results proved in those references. 


\section{Optimality}

Now we construct examples to show the decay rates presented in Theorem 2.4 and Corollary 2.6 are optimal.

Theorem 3.1. Let $\varepsilon>0$ be fixed. If $r \geq 0$ then there exists a $L^{2}$-positive definite kernel $K$ possessing the following features:

(i) $K$ belongs to $W_{2}^{r}$;

(ii) $\mathcal{K}_{0, r}$ is trace-class;

(iii) $\lambda_{n}(\mathcal{K})=o\left(n^{-1-2 r / m}\right)$;

(iv) If $\lim _{n \rightarrow \infty} n^{\varepsilon+1+2 r / m} \lambda_{n}(\mathcal{K})$ exists then it is positive.

Proof. It is enough to consider the kernel having the following condensed spherical harmonic expansion

$$
K(x, y) \sim 1+\sum_{n=1}^{\infty} \frac{N(m, n)}{n^{m(1+\varepsilon)+2 r}} P_{n}^{m}(x \cdot y), \quad x, y \in S^{m} .
$$

and show that it satisfy (i)-(iv).

In a similar way one can prove the following result.

Theorem 3.2. Let $\varepsilon>0$ be fixed. If $r \geq m / 4$ then there exists a $L^{2}$-positive definite kernel $K$ possessing the following features:

(i) $K$ belongs to $W_{2}^{r}$;

(ii) $K_{0, r}$ belongs to $L^{2}\left(S^{m} \times S^{m}\right)$;

(iii) $\lambda_{n}(\mathcal{K})=o\left(n^{-(m+4 r) / 2 m}\right)$;

(iv) If $\lim _{n \rightarrow \infty} n^{\varepsilon+(m+4 r) / 2 m} \lambda_{n}(\mathcal{K})$ exists then it is positive.

Proof. It suffices to consider the kernel $K$ having a condensed spherical harmonic expansion in the form

$$
K(x, y) \sim 1+\sum_{n=1}^{\infty} \frac{N(m, n)}{n^{m(\varepsilon+1 / 2)+2 r}} P_{n}^{m}(x \cdot y), \quad x, y \in S^{m} .
$$

\section{References}

[1] B. Carl; S. Heinrich; T. Kühn, s-numbers of integral operators with Hölder continuous kernels over metric compacta, J. Funct. Anal. 81 (1988), no.1, 54-73.

[2] M. H. Castro; V. A. Menegatto, Eigenvalue decay of positive integral operators on the sphere, Math. Comp. 81 (2012), no. 280, 2303-2317.

[3] M. H. Castro; V. A. Menegatto; A. P. Peron, Integral operators generated by Mercer-like kernels on topological spaces, Coll. Math., 126 (2012), no.1, 125-138.

[4] F. Cobos; T. Kühn, Eigenvalues of integral operators with positive definite kernels satisfying integrated Hölder conditions over metric compacta, J. Approx. Theory 63 (1990), no. 1, $39-55$.

[5] J. B. Conway, "A course in operator theory", Graduate Studies in Mathematics, 21. American Mathematical Society, Providence, RI, 2000.

[6] J. C. Ferreira; V. A. Menegatto, Eigenvalues of integral operators defined by smooth positive definite kernels, Integral Equations Operator Theory 64 (2009), no. 1, 61-81. 
[7] J. C. Ferreira; V. A. Menegatto; A. P. Peron, Integral operators on the sphere generated by positive definite smooth kernels, J. Complexity 24 (2008), no. 5-6, 632-647.

[8] I. C. Gohberg; M. G. Krein, "Introduction to the theory of linear nonselfadjoint operators", Translated from the Russian by A. Feinstein, Translations of Mathematical Monographs, Vol. 18 American Mathematical Society, Providence, R.I., 1969.

[9] I. C. Gohberg; S. Goldberg; N. Krupnik, "Traces and determinants of linear operators", Operator Theory: Advances and Applications, 116. Birkhäuser Verlag, Basel, 2000.

[10] C. W. Ha, Eigenvalue decay of positive definite kernels on the sphere, SIAM J. Math. Anal. 17 (1986), no. 2, 415-419.

[11] Y. B. Han, Singular numbers and eigenvalues of $H^{p}$ kernels, (Chinese) Acta Math. Sinica 34 (1991), no. 1, 118-123.

[12] S. Heinrich; T. Kühn, Embedding maps between Hölder spaces over metric compacta and eigenvalues of integral operators, Indag. Math. 47 (1985), no. 1, 47-62.

[13] H. König, "Eigenvalue distribution of compact operators", Operator Theory: Advances and Applications, 16. Birkhäuser Verlag, Basel, 1986.

[14] T. Kühn, Eigenvalues of integral operators generated by positive definite Hölder continuous kernels on metric compacta, Indag. Math. 49 (1987), no. 1, 51-61.

[15] T. Kühn, Eigenvalues of integral operators with smooth positive definite kernels, Arch. Math. 49 (1987), no. 6, 525-534.

[16] V. A. Menegatto; A. C. Piantella, Convergence for summation methods with multipliers on the sphere, Numer. Funct. Anal. Optim. 31 (2010), no. 6, 738-753.

[17] V. A. Menegatto; A. C. Piantella, Old and new on the Laplace-Beltrami derivative, Numer. Funct. Anal. Optim. 32 (2011), no. 3, 309-341.

[18] A. Pietsch, "Eigenvalues and s-numbers", Cambridge Studies in Advanced Mathematics 13, Cambridge University Press, Cambridge, 1987.

[19] W. Rudin, Uniqueness theory for Laplace series, Trans. Amer. Math. Soc. 68, (1950), 287303.

[20] M. Wehrens, Best approximation on the unit sphere in $\mathbb{R}^{k}$, "Functional analysis and approximation" (Oberwolfach, 1980), pp. 233-245, Internat. Ser. Numer. Math., 60, Birkhäuser, Basel-Boston, Mass., 1981.

[21] M. Wehrens, "Legendre-Transformationsmethoden und approximation von funktionen auf der einheitskugel in $R^{3 "}$, Doctoral Dissertation, RWTH Aachen, 1980. 